\title{
Infoparticip@: periodismo para la participación ciudadana en el control democrático. Criterios, metodologías y herramientas
}

\author{
Amparo Moreno SARDÁ \\ Universidad Autónoma de Barcelona \\ amparo.moreno@uab.cat \\ Pedro Molina RodríGueZ-NAVAS \\ Universidad Autónoma de Barcelona \\ pedro.molina@uab.es \\ Marta CORCOY RIUS \\ Universidad Autónoma de Barcelona \\ marta.corcoy@uab.cat \\ Antonio Aguilar PÉREZ \\ Universidad Autónoma de Barcelona \\ antonio.aguilar@correos.com \\ Miquel Borràs FARRAN \\ Universidad Autónoma de Barcelona \\ miquel@comcom.cat
}

Recibido: 30/10/2012

Aceptado: 04/02/2013

\begin{abstract}
Resumen
El periodismo de información general se ha deshumanizado: ha expulsado a las ciudadanas y los ciudadanos como protagonistas de la democracia y del debate público. Es necesario renovarlo para que recupere una credibilidad que afecta también a la credibilidad de la democracia. Con el proyecto Infoparticip@ (www.infoparticipa.com) nos proponemos colaborar para que adquiera calidad humana a partir de unas fuentes fundamentales como son las administraciones públicas. Utilizamos Internet para facilitar la participación de la ciudadanía plural en el seguimiento y la evaluación de las actuaciones de los responsables políticos y de las políticas públicas. En este artículo explicamos el diagnóstico del que partimos y el trabajo que hemos realizado entre 2008 y 2011 para definir los criterios, las metodologías y las herramientas concebidas para impulsar esta renovación.

Palabras clave: Periodismo, administraciones públicas, participación ciudadana, control democrático, ciudadanía plural.

\section{Infoparticip@: journalism for citizen participation in the democratic control. Criteria, methodologies and tools}

\begin{abstract}
Overview journalism has dehumanized: has expelled the citizens as protagonists of democracy and public debate. It is needed to renew it in order to retrieve its credibility that also affects the credibility of democracy. With the project Infoparticip@ (www.infoparticipa.com) we intend to collaborate to make it acquire human quality based on fundamental sources such as public administrations. We use the Internet to facilitate citizen participation in plural monitoring and evaluation of the actions of policy makers and public policies. This article explains the diagnosis we started from and the work we have done between 2008 and 2011 in order to define the criteria, methodologies and tools designed to foster renewal.
\end{abstract}

Keywords: Journalism, public administration, public participation, democratic control, plural citizenship. 


\section{Referencia normalizada}

MORENO SARDÁ, Amparo; MOLINA RODRÍGUEZ-NAVAS, Pedro; CORCOY RIUS, Marta; AGUILAR PÉREZ, Antonio; y BORRÁS FARRAN, Miquel (2013): "Infoparticip@: periodismo para la participación ciudadana en el control democrático. Criterios, metodologías y herramientas". Estudios sobre el Mensaje Periodístico. Vol. 19, Núm. 2 (julio-diciembre), págs.: 783-803. Madrid, Servicio de Publicaciones de la Universidad Complutense.

Sumario: 1. Deshumanización, hiperhumanización y calidad humana del periodismo. 2. Infoparticip@: información pública para la participación ciudadana en el control democrático. 3. Periodismo para hacer visible la participación de las mujeres en los proyectos de mejora de barrios subvencionados por la Generalitat de Cataluña; 3.1. Primera tarea: localizar y evaluar la información disponible sobre los proyectos de la Ley de Barrios; 3.1.1. Primera fase (2008-2009); 3.1.2. Segunda fase (2010-2011); 3.1.2.1. Análisis de los boletines municipales; 3.1.2.2. Entrevistas con responsables municipales; 3.2. Segunda tarea: elaboración de una herramienta en línea sobre planes de barrio y actuaciones para la equidad entre mujeres y hombres en el uso del espacio y los equipamientos sociales (www.infoparticipa.com/icd); 3.3 . Tercera tarea: definición de criterios y metodologías a seguir para implementar Infoparticip@ en las administraciones públicas; 3.3.1. Cinco criterios básicos para construir información pública periodística con calidad humana; 3.3.2. Propuestas organizativas. 4. Necesidad de una Ley de Transparencia que obligue a las administraciones públicas a ofrecer información que facilite la participación ciudadana en el control democrático. 5. Referencias.

\section{Deshumanización, hiperhumanización y calidad humana del periodismo}

El periodismo de información general, desde la transición, ha ido marginando a las ciudadanas y los ciudadanos como protagonistas y sujetos activos de la actividad política y del debate público. La Constitución Española de 1978 reconoció el derecho de las mujeres a incorporarse a la actividad política y desde entonces buena parte han accedido también al mercado de trabajo y otros escenarios públicos. Sin embargo, los diarios de referencia no han hecho visible esta realidad en la misma proporción. Y no sólo porque han persistido en centrar la atención en los varones adultos de los colectivos dominantes y han mantenido este enfoque que hemos definido como androcéntrico, sino también porque han desplazado la mirada hacia las instituciones, las entidades y los datos abstractos vinculados a los centros de poder (Moreno Sardà, 1998 y 2007), debido a la influencia cada vez mayor de las fuentes interesadas en las rutinas de los profesionales y la definición de la agenda periodística (Tuchman, 1978). En consecuencia, el periodismo se ha deshumanizado y ha dificultado así la participación ciudadana en el control democrático, y la posibilidad de identificar quiénes son los representantes políticos responsables de la gestión de los recursos colectivos. De este modo, ha renunciado a su papel como cuarto poder que debería colaborar al rendimiento de cuentas. Paralelamente, para atraer grandes audiencias, otros medios han sobredimensionado los sentimientos individuales y los han situado en el centro de las conversaciones colectivas, se han hiperhumanizado. El resultado es un ruido mediático que se amplifica en Internet y contamina y obstruye el debate público, entorpeciendo encontrar soluciones para resolver los problemas.

Este diagnóstico, que hemos constatado en los últimos años a partir de varias investigaciones $^{1}$, nos hizo pensar en la necesidad de renovar el periodismo incorporando

1 Moreno Sardà, A. (dir.), La representación de la transformación de las relaciones entre mujeres y hombres en la prensa (1974-2004); y Representación del trabajo de las mujeres en 
calidad humana. Los y las periodistas han de situar en el centro y hacer visibles a las mujeres y los hombres de diferentes edades, procedencias, condiciones sociales, capacidades y necesidades, y han de considerarlos protagonistas de la democracia. Han de producir la información para facilitarles ejercer su derecho a participar en el seguimiento y la evaluación de la acción de los gobiernos y las políticas públicas de forma efectiva. En esta tarea, podíamos utilizar los recursos que ofrece Internet, tal como los habíamos experimentado desde 1998 en el marco de las asignaturas de Historia de la Comunicación ${ }^{2}$, para construir un conocimiento capaz de articular aportaciones colectivas y personales (Moreno Sardà, 2007).

En 2003, a las puertas de las elecciones municipales, estudiantes del Posgrado sobre Producción y Gestión de la Comunicación Local ${ }^{3}$ elaboraron el Decálogo de Buenas Prácticas de la Comunicación Pública Local (http://labcompublica.info/innovacio/decaleg-de-bones-practiques). Y en 2006 reformulamos la metodología que utilizábamos en las investigaciones sobre la prensa para evaluar la mirada informativa (Moreno Sardà, 1998), en el test ADSH, para evaluar la amplitud, la diversidad y la sensibilidad humana de la mirada informativa, y lo presentamos en una Guía para facilitar que cualquier periodista que tuviera interés pudiera modificar este enfoque asumido en las rutinas académicas y profesionales (Moreno Sardà, Rovetto, Buitrago, $2007)^{4}$.

\section{Infoparticip@: información pública para la participación ciudadana en el con- trol democrático}

Estos y otros antecedentes nos condujeron a concebir, en 2007, Infoparticip@ (www.infoparticipa.com) como un prototipo de periodismo para facilitar la participación de la ciudadanía plural en el control democrático. Partíamos de la consideración de que debíamos empezar por revisar el funcionamiento de las administraciones públicas como fuentes de información y de la información que proporcionan los representantes políticos que son responsables. La primera conceptualización de

los medios de comunicación: de la marginación a la utilización de internet 2 para la participación en la construcción de conocimiento y la evaluación de políticas públicas; ambos proyectos I+D financiados por el Instituto de la Mujer en 2001-2004 y 2008-2009, respectivamente. De estos proyectos se han derivado varias Tesis Doctorales y Tesinas que han confirmado estas conclusiones: de San Martín, 2005; Simelio, 2007; Vargas, 2007; Rovetto, 2010. Otros trabajos se centraron en analizar los medios de comunicación municipales: Corcoy et al., 2001; Corcoy, 2012.

2 El curso 1998-1999 construimos la web Paseos por las redes de comunicación desde..., en el Campus Virtual de la Universidad Autónoma de Barcelona, para la docencia de las asignaturas de Historia de la Comunicación. Y en este marco, en 2006 colaboramos en el proyecto liderado por el Ayuntamiento de Tortosa para elaborar el portal Soctortosa.cat, para la participación ciudadana en la construcción de conocimiento colectivo.

3 Producción y Gestión de la Comunicación Local, posgrado impartido por el Laboratorio de Comunicación Pública de la Universidad Autónoma de Barcelona entre 2002 y 2007.

${ }^{4}$ Fem visibles les aportacions de les dones com a ciutadanes actives de les notícies, Proyecto financiado por el Institut Català de les Dones de la Generalitat de Catalunya, 2006-2007. 
Infoparticip@la formulamos en el proyecto que presentamos a la convocatoria del Plan Avanza ${ }^{5}$. Una ayuda del Vicerrectorado de Proyectos Estratégicos de la UAB nos permitió avanzar en el diseño y redimensionar el proyecto. Y en febrero de 2008 presentamos otro proyecto títulado Periodismo para hacer visible la participación de las mujeres en los proyectos de mejora de barrios subvencionados por la Generalitat de Catalunya, que fue financiado por Institut Català de les Dones (ICD) de la Generalitat de Catalunya en dos fases: 2008-2009 y 2010-2011 ${ }^{6}$. Explicamos a continuación los pasos que hemos dado hasta 2012, base del proyecto en el que trabajamos actualmente?

La Ley de Barrios ${ }^{8}$ fue una iniciativa mediante la cual el gobierno de la Generalitat formado por PSC, ERC e ICV decidió conceder ayudas a los ayuntamientos que presentasen un proyecto para mejorar un barrio degradado, problema que afectaba a muchos municipios que difícilmente podían solucionar sólo con recursos propios. Entre 2004 y 2010 se concedieron ayudas para desarrollar 144 planes en 44 municipios. Tras las elecciones de diciembre de 2010, el gobierno de CiU presidido por Artur Mas limitó su aplicación a los proyectos comprometidos.

La Ley de Barrios proporcionaba un campo interesante para poner a prueba el prototipo Infoparticip@. En primer lugar, porque aunque estaba promovida por el Departamento de Política Territorial y Obras Públicas (DPTOP), no se limitaba a promover intervenciones urbanísticas sino que proponía actuaciones integrales que comprendían las condiciones de vida de las mujeres y los hombres a los que debía beneficiar, lo que obligaba a los ayuntamientos a desarrollar un trabajo interdepartamental, transversal, y con participación ciudadana. En su artículo 7.2 establecía que

5 El proyecto se presentó a la convocatoria Plan Avanza, Centros del Conocimiento y Contenidos, del Ministerio de Industria, Turismo y Comercio, con el título: Desarrollo del Laboratorio de Comunicación Pública de la Universidad Autónoma de Barcelona como centro experimental de periodismo para la participación ciudadana, y del prototipo web Infoparticip@. De acuerdo con la resolución de diciembre de 2007 (Exp. No: PAV-100000-2007-58), se concedió un presupuesto financiable de 1.011.031 euros, de los cuales 141.031 euros en forma de subvención y 870.000 euros en forma de préstamo. Ante la entonces incipiente crisis económica consideramos inviable poder retornar el crédito y decidimos renunciar.

6 Convocatorias del Institut Català de les Dones 2008-2009 y 2010-2011.

7 Se trata del Mapa de las Buenas Prácticas de la Comunicación Pública Local (www.infoparticipa.com/bones-practiques), que hemos desarrollado en Cataluña desde febrero de 2012 y que extenderemos a otras 5 comunidades autónomas de España entre 2013 y 2015 gracias al proyecto $\mathrm{I}+\mathrm{D}+\mathrm{I}$ titulado Comunicación y periodismo para la participación ciudadana en el seguimiento y la evaluación de la gestión de los gobiernos locales, concedido por el Ministerio de Economía y Competitividad (referencia CSO2012-34687). En este proyecto colaboramos 7 universidades de 6 comunidades autónomas. La herramienta dispone de un gestor de contenidos muy ágil que permite trabajar de forma cooperativa y en red. Está previsto extenderlo también a Iberoamérica.

8 Llei 2/2004, de 4 de juny, de millora de barris, àrees urbanes $i$ viles que requereixen una atenció especial, publicada en el DOGC 4151, de 10/06/2004. Texto de la Ley en: http://www.gencat.cat/diari/4151/04154100.htm 
los proyectos debían prever varios tipos de actuaciones, entre otras, intervenciones orientadas a la equidad entre mujeres y hombres, o equidad de género, en el uso del espacio urbano y los equipamientos, por tanto, obligaba a tener en cuenta a la pluralidad de mujeres y hombres9. Un Manual para la elaboración de los proyectos de intervención integral en los barrios y áreas urbanas de atención especial y para la elaboración del Informe de Evaluación Final, aclaró el significado y el alcance de la noción de equidad de género en el uso del espacio urbano y los equipamientos:

"La perspectiva de género en urbanismo propone redefinir los objetivos del urbanismo según las necesidades de la nueva sociedad, basada en la igualdad de oportunidades entre hombres y mujeres, entre generaciones y entre culturas. Aplicar la perspectiva de género en el diseño de las ciudades y pueblos significa hacer cambios en los planteamientos del urbanismo actual, introduciendo en la agenda de prioridades temas importantes de la vida cotidiana como la seguridad, la proximidad, el tiempo y la organización los servicios, así como la mezcla de usos que ayude a cubrir la diversidad de actividades que actualmente llevan a cabo en gran medida las mujeres y que en el futuro se repartirán entre mujeres y hombres" (Generalitat de Catalunya. Departament de Política Territorial i Obres Públiques, 2010: 18$)^{10}$

En este Manual se definieron unos campos de actuación y se propusieron ejemplos de indicadores para evaluar el resultado / impacto de estas actuaciones:

Actuación 6.1. Creación y mejora de espacios seguros, vivos y amigables.

Actuación 6.2. Programas y talleres de participación en el diseño de las ciudades bajo la perspectiva de género.

Actuación 6.3. Incorporación de criterios de proximidad en el espacio público y los equipamientos (Generalitat de Catalunya. Departament de Política Territorial i Obres Públiques, 2010: 18).

Para que sean eficaces, estas formulaciones políticas, que incorporan a las mujeres como protagonistas de las intervenciones políticas y han generado propuestas legales y recursos económicos, han de contar con unos medios de comunicación que las den a conocer y faciliten la participación de las ciudadanas y los ciudadanos (MartíCosta et alt., 2009). Por tanto, este marco nos permitía trabajar en una doble dirección: a) contrastar actuaciones previstas en unos planes aprobados y valoradas en unos pre-

9 La Ley incorporaba, así, los principios del V Plan de Acción y Desarrollo de las Políticas de Mujeres (2005-2007) (Generalitat de Catalunya, Institut Català de les Dones, 2006) que definía como primer objetivo implementar las políticas de las mujeres como un eje transversal en el diseño y desarrollo de las políticas del Gobierno. Este criterio de transversalidad implicó incorporar las aportaciones de las mujeres en los diferentes ámbitos de actuación y en todas las políticas del Gobierno de la Generalitat, y promover medidas específicas dirigidas a las mujeres desde todas sus áreas. Esta orientación política tuvo continuidad en el Plan de Políticas de Mujeres del Gobierno de la Generalitat de Cataluña (2008-2011) (Generalitat de Catalunya; Institut Català de les Dones, 2008).

${ }^{10}$ Indicación de fuente en el documento: "Diputació de Barcelona. Extret de les conclusions de les Jornades d'urbanisme i gènere". La traducción de este y del resto de textos del catalán al castellano han sido hechas por los propios autores. 
supuestos, con las informaciones proporcionadas por las administraciones municipales, informaciones que necesariamente debían hacer visibles a las mujeres; y b) tras identificar las aportaciones y las deficiencias, plantearnos cómo construir la información de manera que facilitase la participación de la ciudadanía plural en el control de las administraciones públicas tanto locales como supralocales, ya que la Generalitat de Catalunya, a su vez, gestionaba fondos europeos.

\section{Periodismo para hacer visible la participación de las mujeres en los proyectos de mejora de barrios subvencionados por la Generalitat de Catalunya}

El proyecto que propusimos contemplaba estas tareas: localizar y evaluar la información disponible sobre los proyectos de la Ley de Barrios proporcionada por el Departamento de Política Territorial de la Generalitat y por los ayuntamientos que habían recibido subvenciones; y definir criterios y metodologías para desarrollar herramientas y modelos que permitieran hacer visible e inteligible la información necesaria para poder evaluar las actuaciones.

\subsection{Primera tarea: localizar y evaluar la información disponible sobre los pro- yectos de la Ley de Barrios}

Como hemos explicado, realizamos este proyecto en dos fases: en la primera (2008 y 2009), nos centramos en localizar y evaluar la información disponible, y construimos una herramienta en línea para presentarla; y en la segunda (2010 y 2011), completamos y actualizamos el diagnóstico y la publicación de la información disponible, y elaboramos en un manual los criterios y las metodologías a seguir para poder implementar el prototipo Infoparticip@ en las administraciones públicas.

\subsubsection{Primera fase (2008-2009)}

El primer paso fue estudiar la Ley de Barrios y otros documentos necesarios para comprender su ejecución, especialmente en lo relativo al punto 6 sobre equidad de género en el uso de los espacios urbanos, y sobre políticas de igualdad de la Generalitat de Catalunya. Apreciamos así, la dificultad que supone comprender la planificación urbana desde una perspectiva no-androcéntrica dados los hábitos de pensamiento aprendidos y reproducidos en la actividad profesional, que impiden contemplar los problemas y sus soluciones teniendo en cuenta la pluralidad social.

Para clarificar esta cuestión resultaron de gran ayuda las publicaciones de Anna Bofill (2005 y 2008) y de Zaida Muxí (2009a, 2009b y Muxí y Casanovas, 2009), sobre planeamiento urbanístico y vivienda recogiendo las necesidades de las mujeres, y los trabajos de Isabel Segura (1995 y 1998) que abordan la historia de las ciudades integrando las aportaciones de las mujeres. Advertimos que al ampliar el punto de vista convencional, androcéntrico, para incorporar las aportaciones de las mujeres, se integran también las necesidades de otros seres humanos y se abre un abanico más amplio de experiencias y posibilidades.

El paso siguiente fue averiguar qué información proporcionaban las administraciones públicas implicadas en los planes de barrio y concretamente sobre las actuaciones relacionadas con el punto 6. Para ello, analizamos las páginas webs del 
Departamento de Política Territorial y Obras Públicas de la Generalitat y de los ayuntamientos con planes aprobados en las tres primeras convocatorias (total 47), y los boletines municipales. Concluimos que se estaba ofreciendo una comunicación insuficiente y deficiente: solamente en 10 de las webs municipales se hablaba de actuaciones con perspectiva de género y tan solo en dos se daban datos sobre los presupuestos. En cuanto a los boletines municipales, revisamos 1.760 ejemplares en los que encontramos 110 noticias sobre los planes de barrio, de las cuales únicamente el 9.2\% hacían visibles a las mujeres o las actuaciones con perspectiva de género. En los boletines específicos sobre los planes de barrios este porcentaje se reducía al 8,7\%. Solo en las dos publicaciones dirigidas a las mujeres éstas eran visibles en el 100\% de las noticias publicadas.

Para completar esta información, diseñamos y realizamos un cuestionario que enviamos a todos los ayuntamientos con planes aprobados. La ficha contenía una primera parte con datos de identificación del plan, y una segunda con 15 preguntas. Recibimos 30 respuestas que nos aportaron más información y la visión del personal técnico. Establecimos así unas conclusiones y recomendaciones:

- Los municipios deben contar con un plan de comunicación para el plan de barrios que debe contemplar formas de participación adecuadas a los diferentes perfiles de las ciudadanas y los ciudadanos.

- Los equipos técnicos deben trabajar transversalmente y contar con especialistas en igualdad.

- La participación de las mujeres en las actuaciones debe medirse y comunicarse.

- Las asociaciones y grupos de mujeres deben estar presentes en los órganos de decisión y seguimiento.

- Las informaciones deben redactarse haciendo visibles a las mujeres, las situaciones de discriminación, y dándoles voz, utilizando un lenguaje inclusivo sin dificultar la lectura.

Estos resultados nos hicieron pensar que debíamos profundizar en la noción de qué se considera información pública, noticia y fuente de información pública, y en el papel que desempeñan los gabinetes de comunicación de los ayuntamientos.

\subsubsection{Segunda fase (2010-2011)}

En la segunda fase del proyecto nos propusimos completar la evaluación de la información pública municipal sobre los planes de barrio con un análisis detallado de noticias publicadas en boletines municipales, y realizando entrevistas a responsables de la comunicación y de los planes para identificar las rutinas de trabajo que condicionan la información que ofrecen.

Por una parte, realizamos un examen minucioso de las rutinas y los recursos que utilizan los gabinetes de comunicación de las administraciones públicas para definir qué consideran noticia, y concretamente, para valorar como noticias las actuaciones de las mujeres. Este examen se basó en un análisis de las informaciones publicadas, y se completó con entrevistas a personas responsables de la comunicación de algunos ayuntamientos, para profundizar en las rutinas que siguen en su proceso de trabajo y 
determinar los procedimientos que ayudarían a mejorar la información y la comunicación a la ciudadanía plural.

Para evaluar cómo representaban los boletines municipales a las mujeres de diferentes edades, condiciones sociales y procedencias como protagonistas activas de los planes de barrios, aplicamos el Test ADSH, para evaluar la Amplitud, la Diversidad y la Sensibilidad Humana de la mirada informativa, que habíamos formulado a partir de investigaciones anteriores (Moreno Sardà et al., 2007) que plantea cinco interrogantes: ¿quién enfoca? ¿a quién? ¿haciendo qué? ¿en qué escenarios? y ¿utilizando qué fuentes?

Para realizar las entrevistas elaboramos un cuestionario, si bien se buscó también el diálogo con los y las participantes para aprovechar las diferentes perspectivas en función de sus responsabilidades, como profesionales de la comunicación o como personal técnico de otras áreas municipales.

En ambos trabajos se tomaron como muestra los 5 municipios que en la primera fase del proyecto consideramos ejemplos de buenas prácticas (Granollers, El Prat, Lleida, Sant Cugat y Montcada i Reixac), con planes aprobados en diferentes convocatorias (uno de ellos, Lleida, con 3, y otro, Montcada i Reixac, con 2), con importantes concentraciones de población, gobernados por diferentes fuerzas políticas, situados en cuatro comarcas diferentes y dos de ellos capitales comarcales (Granollers y Lleida). También tuvimos en cuenta las facilidades que dieron para hacer las entrevistas.

\subsubsection{Análisis de los boletines municipales:}

El análisis de las noticias publicadas en los boletines municipales de estos ayuntamientos, desde septiembre de 2009 hasta octubre de 2011, proporcionó los siguientes resultados.

Tabla 1. Protagonistas en los titulares de las noticias analizadas. Elaboración propia.

\begin{tabular}{|l|c|c|}
\hline \multicolumn{1}{|c|}{ Humanos } & Valores absolutos & Porcentajes \\
\hline Mujeres identificadas con nombres y apellidos & 3 & $3 \%$ \\
\hline Mujeres sin identificar & 0 & $0 \%$ \\
\hline Hombres identificados con nombres y apellidos & 0 & $0 \%$ \\
\hline Hombres sin identificar & 0 & $0 \%$ \\
\hline Colectivos No humanos & 7 & $7 \%$ \\
\hline Total humanos & 10 & $10 \%$ \\
\hline \multicolumn{1}{|c|}{} & 12 & $12 \%$ \\
\hline Instituciones & 76 & $78 \%$ \\
\hline Otros & $\mathbf{8 8}$ & $\mathbf{9 0 \%}$ \\
\hline Total no humanos & $\mathbf{9 8}$ & $\mathbf{1 0 0 \%}$ \\
\hline
\end{tabular}


Tabla 2. Protagonistas en los elementos gráficos de las noticias analizadas. Elaboración propia.

\begin{tabular}{|l|c|c|}
\hline \multicolumn{1}{|c|}{ Humanos } & Valores absolutos & Porcentajes \\
\hline Mujeres identificadas con nombres y apellidos & 17 & $9 \%$ \\
\hline Mujeres sin identificar & 39 & $21 \%$ \\
\hline Hombres identificados con nombres y apellidos & 17 & $9 \%$ \\
\hline Hombres sin identificar & 43 & $24 \%$ \\
\hline Colectivos No humanos & 3 & $2 \%$ \\
\hline Total humanos & 119 & $65 \%$ \\
\hline & & $0 \%$ \\
\hline Instituciones & 0 & $35 \%$ \\
\hline Otros & 64 & $35 \%$ \\
\hline Total no humanos & 64 & $100 \%$ \\
\hline Total protagonistas & 183 & \\
\hline
\end{tabular}

Tabla 3. Protagonistas en el cuerpo de las noticias analizadas. Elaboración propia.

\begin{tabular}{|l|c|c|}
\hline \multicolumn{1}{|c|}{ Humanos } & Valores absolutos & Porcentajes \\
\hline Mujeres identificadas con nombres y apellidos & 28 & $4 \%$ \\
\hline Mujeres sin identificar & 5 & $1 \%$ \\
\hline Hombres identificados con nombres y apellidos & 46 & $8 \%$ \\
\hline Hombres sin identificar & 5 & $1 \%$ \\
\hline Colectivos No humanos & 178 & $30 \%$ \\
\hline Total humanos & 262 & $44 \%$ \\
\hline \multicolumn{1}{|c|}{} & 163 & $28 \%$ \\
\hline Instituciones & 163 & $28 \%$ \\
\hline Otros & $\mathbf{3 2 6}$ & $\mathbf{5 6} \%$ \\
\hline Total no humanos & $\mathbf{5 8 8}$ & $\mathbf{1 0 0 \%}$ \\
\hline & &
\end{tabular}

Estos datos permiten extraer las siguientes conclusiones:

1. En los titulares (Tabla 1), predominan los protagonistas no humanos $(88 ; 90 \%$; instituciones 12 , otros 76) y los protagonistas humanos son minoritarios (10, $10 \%$; colectivos 7 ; mujeres identificadas 3 ).

2. En los elementos gráficos (Tabla 2), se enfoca más a los protagonistas humanos $(119,65 \%$; 43 hombres sin identificar y 39 mujeres sin identificar; 17 hombres y 17 mujeres identificadas, 3 colectivos) y son minoría los protagonistas no humanos (64, $35 \%$; ninguna institución; todos "otros").

3. En el cuerpo de las noticias (Tabla 3), la presencia de los protagonistas es más equilibrada. Sin embargo, la de los protagonistas humanos es inferior (262; $44 \%$, colectivos 178 , hombres identificados 46 , mujeres identificadas 28 ; hombres y mujeres no identificadas, 5) a la de los protagonistas no humanos (326; $56 \%$, que se distribuyen en la misma cantidad los que son instituciones y otros).

Se confirma así que las rutinas de los profesionales favorecen la deshumanización del periodismo

\subsubsection{Entrevistas con responsables municipales}

En cuanto a las entrevistas, el tema central fue el flujo y tratamiento de la información en los medios municipales, concretándose en cuestiones relevantes en torno al trata- 
miento de la información sobre los planes de barrio y específicamente sobre las actuaciones con perspectiva de género. Tras la reflexión a que obliga una entrevista, se preguntaba qué problemas se consideraban más importantes y sobre qué proponían mejoras. También se interrogaba sobre algunos ejemplos de las propias publicaciones municipales, lo que permitió reafirmar o apreciar contradicciones entre los resultados del análisis y las respuestas.

Las entrevistas se transcribieron completas y los resultados se sistematizaron en 10 puntos:

1) Ciudadanía plural. En algunos casos se revisan las informaciones e imágenes para que estén representados los diferentes colectivos y otros consideran que se deben seguir las directrices políticas. Para visibilizar diferentes temas y colectivos se debe dar importancia a la información proveniente de las diferentes concejalías y entidades.

2) Representantes políticos paritarios. Se valora como ejemplar la representación política paritaria tanto en el equipo de gobierno como en la oposición.

3) Plan de barrios. La comunicación sobre el plan de barrios debe contribuir a normalizar la imagen de los barrios degradados y facilitar la implicación del vecindario en los proyectos. La proximidad, mediante oficinas de información y otras estrategias, se consideran buenas prácticas. En algunos municipios se han hecho publicaciones específicas y se ha ido informando con continuidad; otros lo han hecho con más dificultades y demasiado centrados en las obras; pocas veces se informa de las actuaciones realizadas con perspectiva de género, cuestión relegada a las memorias. Los diferentes planes han impulsado la creación de grupos de mujeres y la participación de otros colectivos de vecinos.

4) Servicio de comunicación e información. Los departamentos de prensa y comunicación dependen de la alcaldía lo que supone un control político muy cercano. También existen empresas municipales que se encargan de los medios. En general, las diferentes concejalías proponen noticias que los departamentos de prensa revisan. Los procedimientos no siempre están formalmente establecidos. Se manifiesta mucha preocupación por atender a todas las fuentes de información. Se prioriza a los políticos y nunca a los técnicos. En algunos casos se diferencia netamente entre medios que dan información de la gestión y otros más generalistas donde sí se contrastan más las informaciones. El término "transparencia" no es utilizado de forma generalizada. En cuanto a los destinatarios de la información, se considera a la población adulta sin más diferencias. A menudo se pone en duda la pertinencia de un enfoque para mujeres.

5) Actuaciones sobre las que se da información. Está asumido que se debe dar información sobre la gestión y el seguimiento del PAM, pero menos sobre el presupuesto y el Pleno Municipal. Los documentos técnicos, jurídicos, presupuestos, etc., se cuelgan en las webs sin elaborar ya que se considera que la información institucional no es susceptible de interpretaciones. Se hacen reuniones con las entidades para explicar los presupuestos municipales u otros grandes asuntos. Algunos ayuntamientos entienden que el departamento de comunicación debe informar de todas las actuaciones desde que se proyectan, por eso multiplican las notas de prensa y uti- 
lizan monográficos y buzoneo. Los plenos municipales se consideran poco interesantes porque no es el lugar donde se generan los grandes debates, como tampoco lo es la junta de gobierno. Admiten que no conocen ciertas informaciones, como las que pueden denotar desacierto en la gestión, y por tanto no pueden informar.

6) Información y participación ciudadana. La participación ciudadana no parece una prioridad, dada la falta, en general, de respuestas interesantes. Las nuevas tecnologías se ven como aliadas. En algún caso se utiliza la televisión para que la ciudadanía pueda preguntar directamente al alcalde o alcaldesa sobre cualquier asunto. Los boletines se ven como elementos cohesionadores y, a la vez, medios para difundir actos y propiciar una mayor participación.

7) Información con perspectiva de género. En general, se tiene en cuenta que se debe dar visibilidad a mujeres y a hombres pero se reiteran los comentarios que afirman que es muy importante la formación y haber incorporado esta perspectiva de trabajo. El lenguaje inclusivo no está plenamente incorporado. Se da relevancia en fechas señaladas como el 8 de marzo. Algún municipio organiza talleres de comunicación para que las mujeres ganen confianza y presencia en los medios.

8) Medios de comunicación locales privados. Se pone de manifiesto el interés por facilitarles información, al tiempo que una cierta decepción porque a menudo reproducen los comunicados sin contrastar la información. Existen convenios con televisiones privadas. También existen publicaciones vecinales con tradición con las que se mantienen diferentes formas de colaboración y que pueden ser subvencionadas, pero cada vez más se tiende a las publicaciones digitales. Cabe destacar la nula presencia de publicaciones de mujeres, aunque algunas organizaciones disponen de espacio en la web municipal.

9) Representantes de la oposición. Se pone de manifiesto la necesidad de contar con normativas que regulen la participación y presencia de los partidos políticos en los medios de comunicación municipales.

10) Cómo mejorar la comunicación pública local. En general, los profesionales entrevistados se manifiestan satisfechos con los resultados de su trabajo. Algunos manifiestan que les inquieta la falta de tiempo para reflexionar y para revisar. En algunos casos se cuenta con consejos de redacción en los que participan técnicos municipales. A veces, los políticos son la fuente de información, cuando los técnicos de las diferentes áreas podrían dar una información más completa. También se pide a los políticos más respeto por los profesionales, en el sentido de que deberían dejarse aconsejar. Hay conciencia casi generalizada de la insuficiente utilización del lenguaje inclusivo y la necesidad de hacer informaciones incorporando a otros sectores de la ciudadanía que generalmente quedan excluidos, como residentes de distritos alejados de los centros o inmigrantes, mujeres, etc. También se habla de la dificultad de visibilizar a las mujeres cuando se trabajan temas calificados como "duros" como urbanismo o economía.

\subsection{Segunda tarea: elaboración de una herramienta en línea para informar sobre planes de barrio y actuaciones para la equidad entre mujeres y hombres en el uso del espacio y los equipamientos sociales (www.infoparticipa.com/icd)}


Para contrarrestar la información insuficiente y deficiente ofrecida en los medios de comunicación de las administraciones públicas municipales y de la Generalitat, decidimos elaborar una herramienta en línea que nos permitiese publicar los resultados de nuestra investigación así como los datos obtenidos sobre los planes de barrio, y experimentar con las posibilidades que ofrece la Web 2.0. Esto nos ayudaría a sentar las bases para diseñar e implementar el prototipo Infoparticip@.

Las fuentes que utilizamos fueron las webs institucionales, las respuestas al cuestionario que enviamos en la primera fase a los técnicos vinculados a los planes de barrios, y los resultados de las entrevistas que realizamos en la segunda fase. Además, el Departamento de Política Territorial y Obras Públicas de la Generalitat de Catalunya nos permitió acceder a los proyectos presentados por los ayuntamientos y ésta fue la fuente principal que utilizamos para completar la información de la herramienta en línea. Actualizamos los datos sobre los planes de barrio subvencionados hasta la convocatoria 2010, en total 149, examinando el apartado "Descripción de la población" para conseguir datos sobre hombres y mujeres residentes en cada barrio, aunque este dato no se ha podido incluir en todos los casos porque no se incorpora en todas las memorias.

Para organizar la información utilizamos los criterios que habíamos definido y probado en la web Paseos por las redes de comunicación desde...11, que habíamos elaborado el curso 1998-1999 en el Campus Virtual de la UAB para la docencia de las asignaturas de Historia de la Comunicación, y que habíamos transformado en una wiki: tal como se explica en la Guía de la misma (https://wikis.uab.cat/passeigperlesxarxes/index.php/La_Guia), se utiliza una base de datos organizada a partir de las coordenadas espacio y tiempo para articular las explicaciones sobre las permanencias y los cambios locales y supralocales, teniendo en cuenta que cada lugar está traspasado por redes de transporte y comunicación que articulan las relaciones personales y colectivas en tramas históricas (Massey, 1994 y 2004). Esta estructura permite organizar una explicación sobre la construcción histórica de la sociedad red desde las distintas posiciones ocupadas por cada persona en cada localidad (Haraway, 1991; Femenias y Soza, 2011), relacionar las experiencias e intereses personales con los contextos históricos más amplios y construir un diálogo y un conocimiento plural. La utilizamos también en el Mapa interactivo de los medios de comunicación en Cataluña (http://labcompublica.info/innovacio/mapa-de-mitjans-de-catalunya) ${ }^{12}$.

Optamos, así, por organizar la información obtenida sobre los planes de barrio en una herramienta en línea en la que vinculamos bases de datos, una representación cartográfica, una línea de tiempo y otras representaciones gráficas. De este modo, podemos georeferenciar la información, esto es, localizarla sobre un mapa (Jiménez Chávez, 2010 y 2012) -en este caso utilizamos Google Map- que permite hacer visibles los planes de barrio concedidos por la Generalitat de Cataluña a cada municipio; ver su distribución por todo el territorio de Cataluña o por comarcas; ver en qué año se concedieron; localizar cada plan en el correspondiente municipio e incluso hacer

${ }^{11}$ Ver nota 2 y https://wikis.uab.cat/passeigperlesxarxes/index.php/P\%C3\%A0gina_principal

${ }^{12}$ Mapa elaborado en 2007-2008 gracias a una ayuda del Consejo Social de la UAB y que desde entonces mantenemos actualizado con el apoyo de la Secretaria de Comunicación de la Generalitat de Catalunya. 
un zoom e identificar el perímetro de la zona afectada -técnicamente podríamos aproximarnos a cada actuación-; acceder a una ficha con diversos datos sobre el municipio, el proyecto, las actuaciones previstas para la equidad entre mujeres y hombres en el uso del espacio, y los recursos de comunicación. También podemos cronorreferenciar la información, es decir, situar los datos en una línea de tiempo en la que se puede representar la duración prevista de cada actuación y a la que se puede asociar documentos diversos. En nuestro caso nos limitamos a ubicar la primera noticia que se publicó en el boletín municipal sobre el plan de barrios y vincular el documento (en formato pdf) con la información, para mostrar las posibilidades que se podrían utilizar. Y podemos, así mismo, utilizar otros recursos gráficos para representar informaciones complejas (Marín Ochoa, 2009); en nuestro caso, los utilizamos para representar datos numéricos, hacer comparaciones entre los proyectos y evaluar la distribución de los recursos atribuidos por la Generalitat por el territorio de Cataluña.

De este modo, cualquier persona interesada que acceda (www.infoparticipa.com /icd) puede elegir ver la información vinculada con el espacio, con el tiempo o representada en gráficos. Si continuamos en la opción espacio, que se abre por defecto, podemos elegir, a partir de las ventanas de la izquierda, la localidad sobre la que queremos tener información, y al hacer clic en una, como por ejemplo Badalona, veremos sobre el mapa una pequeña ficha que dice que este municipio dispone de dos planes de barrio, y que uno cuenta con presupuesto para intervenciones de género; al mismo tiempo, en la ventana de la derecha aparece el nombre de los dos planes y la fecha en que se concedieron (Serra d'en Mena, 2004 y Sant Roc, Artigas, 2006) con unas marcas que indican si el plan tiene o no tiene presupuesto para intervenciones de género; si hacemos clic sobre uno de los planes se abre, sobre el mapa de la localidad, una ficha que ofrece, en la parte superior, cuatro pestañas que permiten acceder a datos generales, una descripción del plan, datos sobre las intervenciones de género y otros sobre los medios de comunicación del ayuntamiento; en todos los casos se indica la fuente de la que proceden; $y$ en la parte inferior de la ficha dos botones permiten ver el perímetro del plan sobre el mapa del municipio, imprimir la ficha o cerrarla. Si pasamos a la pestaña tiempo, vemos una barra que recorre los cuatro años de duración de cada uno de los planes del municipio, en la que en este momento sólo se ha hecho una señal que indica cuando se publicó la primera noticia sobre el plan de barrio en el boletín municipal y da acceso al pdf con el boletín. Si a continuación queremos ver los datos numéricos en forma de gráficos (sección o barras) podemos seleccionar alguna de las preguntas que se han formulado. Lógicamente, todos los resultados responden a la elección realizada al principio, un municipio, los planes de una comarca, los de un año... Esto es sólo un desarrollo para probar y mostrar las posibilidades que ofrecen estas herramientas y las formas de utilizarlas.

En la segunda fase del proyecto completamos la información de la herramienta, actualizamos los datos correspondientes a los planes que se concedieron en los últimos años, y mejoramos las herramientas digitales ${ }^{13}$.

${ }^{13}$ Los detalles se pueden consultar en las respectivas memorias justificativas: http://labcompublica.info/wp-content/uploads/2012/09/Memoria_LCP_ICD_PlaBarris_2003_2007.pdf y http://labcompublica.info/wp-content/uploads/2012/10/Memoria_LCP_ICD_PlaBarris_2010_2011.pdf. 
En definitiva, la herramienta en línea elaborada permite dar cuenta de forma sencilla e inteligible de informaciones complejas, locales y supralocales, desde la proximidad en la que se desarrolla la vida de cada persona y de manera que se pueden compartir y contrastar informaciones y experiencias, y construir conocimiento desde distintas posiciones sociales, en red y de forma colaborativa.

\subsection{Tercera tarea: definición de criterios y metodologías a seguir para imple- mentar Infoparticip@en las administraciones públicas}

Ciertamente, esta herramienta en línea no corresponde completamente a lo que ha de ser Infoparticip@, pero permite mostrar los criterios básicos y los recursos que se pueden utilizar. Ahora bien, para que las administraciones públicas asuman que deben proporcionar una información pública de calidad humana, transparente, potable y comprensible a la ciudadanía plural, es preciso introducir cambios en los flujos de información interna, como hemos constatado al realizar entrevistas a los responsables que, sólo así, dispondrán por su parte de una fuente de información de calidad sobre el comportamiento de los actores implicados en la ejecución de las actuaciones de los gobiernos. Y para ello, es imprescindible empezar por clarificar qué se entiende por información pública o noticia, y qué papel corresponde a las administraciones públicas como fuentes de información de otros medios de comunicación o que se dirigen directamente a la ciudadanía.

Por todo ello nos propusimos elaborar un manual que ayudara a producir y gestionar la información de las administraciones públicas para facilitar una participación de la ciudadanía plural en el control democrático bien fundamentada (Brugué y Gomà, 1998). Para ello, nos inspiramos en los planteamientos que han orientado la reingeniería de procesos para implantar el gobierno electrónico y en procedimientos que se utilizan para la rendición de cuentas en las administraciones y en las empresas (Comision de las Comunidades Europeas, 2001; Freixes y Remotti, 2002; Rose-Ackerman, 2005; Macagnam, 2006; Rivero Menéndez et alt., 2007; Villoria Mendieta y Wences, 2010; Villoria Mendieta 2011).

Coincidimos con Rivero Menéndez, Mora Agudo y Flores Ureba (2007: 2) cuando afirman que "tradicionalmente la rendición de cuentas en el entorno público (...) reducía la obligación de la administración a dar cuenta de su gestión mediante la presentación de las cuentas anuales". Pero recientemente se ha ampliado este enfoque "incorporando una dimensión social (...) defendiendo el derecho de los ciudadanos a ser informados, no sólo de los resultados contables de la entidad, sino también de los objetivos que éstas pretenden alcanzar y de las estrategias que desarrollarán para lograrlos". Y está claro que Internet "facilita la accesibilidad de esta información permitiendo su universalización".

A continuación, exponemos los criterios y las propuestas organizativas y modelos de trabajo que consideramos imprescindibles para implementar Infoparticip@, que desarrollamos en el manual que hemos elaborado y se encuentra en proceso de revisión previo a su publicación. 


\subsubsection{Cinco criterios básicos para construir información pública periodística con calidad humana}

Hemos definido cinco criterios básicos para construir una información pública periodística de calidad, que resulte inteligible a la ciudadanía plural y le facilite una participación efectiva en el seguimiento y la evaluación de la acción de los representantes políticos, en el gobierno y en la oposición:

1) Humanizar la información:

Hay que considerar a las ciudadanas y los ciudadanos como sujetos activos del debate público y la participación democrática, con distintas capacidades y necesidades, e incorporarlos como protagonistas activos de las noticias evitando la despersonalización. También hay que presentar a los y las representantes políticos como sujetos activos que asumen responsabilidades en la gestión pública de los recursos colectivos y han de rendir cuentas.

2) Información completa y transparente:

En un Estado de derecho, la información sobre la gestión política ha de incorporar los procedimientos que la ley marca para llevar a cabo cualquier actuación, de manera que se garantice la supeditación de todos los actores a las reglas de juego colectivas que se han definido democráticamente.

3) Información con memoria y contextualizada:

Las noticias han de tener memoria. Cada hecho que se pueda considerar noticia debe poder evaluarse en relación con las actuaciones del programa de un gobierno. Y esta memoria debe ser lo más completa posible y ha de situarse en el contexto histórico del municipio, para que la cada persona pueda tener una perspectiva que no quede reducida a cada instante y a cada mandato.

4) Información contrastada y contrastable:

Hay que ofrecer todos los datos y documentos que permiten contrastar los pasos que marca la ley para ejecutar cada una de las actuaciones, y las agendas de trabajo de los representantes políticos, para contrastar las palabras con las actuaciones.

5) Información periodística, inteligible y comprensible:

Hay que elaborar la información con criterios periodísticos, adecuarla a las diferentes capacidades y necesidades de la población y producirla de manera que resulte inteligible y comprensible a la ciudadanía plural. No es suficiente con publicar documentos en pdf, ni con acumular datos abiertos (open data).

\subsubsection{Propuestas organizativas}

Para poder aplicar estos criterios es imprescindible modificar el organigrama habitual en las administraciones públicas en relación con los gabinetes de prensa o comunicación: hacer un replanteamiento de esa mezcla, a veces confusa, de funcionalidades que aparecen en muchos planes de comunicación institucionales, entre un periodismo escasamente fundamentado y el marketing político. Nuestra propuesta se centra en la información que se ha de proporcionar a través de la web corporativa porque consideramos que es el soporte más adecuado para la producción, gestión, acumulación y difusión de la información de una administración pública que además puede relacionarse con otras administraciones e incorporar instrumentos de participación ciuda- 
dana. Pero para ello, proponemos introducir algunos cambios en el organigrama actual:

1) Transformar los actuales gabinetes de comunicación e información de las administraciones públicas en la parte más visible (front-office) de un Servicio de Información y Comunicación (SIC).

A menudo, las informaciones elaboradas por los periodistas de fuentes, responsables de los gabinetes de comunicación públicos, no son suficientemente transparentes y completas porque cada servicio técnico y cada concejalía gestiona, según rutinas anquilosadas, los datos que podrían permitir hacer un seguimiento de las actuaciones que realizan. Y el problema con que se encuentran estos profesionales que han de actuar como fuentes para otros medios de comunicación, es que ellos mismos no suelen tener acceso a las fuentes primarias, a los datos que manejan los técnicos responsables de gestionar cada una de las actuaciones: estos periodistas no siempre disponen de la información que necesitarían para cumplir correctamente su tarea. Es necesario, por tanto, reorganizar los flujos de información internos de cada uno de los departamentos o servicios y del conjunto, de manera que confluyan en un Servicio de Información y Comunicación (SIC) para la participación ciudadana efectiva, que garantice la calidad humana de la información que proporcionan las administraciones públicas.

Este SIC debe abarcar dos campos de actuación, de acuerdo con la terminología utilizada en la administración electrónica: a) el back-office, ámbito donde se organizan los flujos de información internos de cada servicio y concejalía, de forma transversal y transparente, para que el conjunto proporcione una información de calidad, transversal, que permita hacer seguimiento del estado de ejecución de cada actuación y del conjunto del presupuesto público; y b) el front-office, el lugar donde se procesa esta información para hacerla inteligible según las capacidades y las demandas específicas de cada ciudadana o ciudadano, o para proporcionarla a los medios de comunicación públicos o privados.

2) Elaborar un Plan de Información y Comunicación para la participación ciudadana efectiva:

La actividad del Servicio de Información y Comunicación (SIC) debe definirse en un Plan de Información y Comunicación que debe ser elaborado al principio de cada mandato y para los cuatro años siguientes, como uno de los compromisos del Plan de Gobierno o Plan de Actuación Municipal (PAM). Periódicamente y más aún al final del mandato, el Consejo del SIC debe hacer un balance del trabajo realizado e incluirlo en el balance de la gestión del gobierno. En este Plan de Información y Comunicación se incluirán intervenciones relacionadas con la participación ciudadana.

3) Elaborar informaciones permanentes y puntuales sobre las actuaciones de los representantes políticos:

La información de las administraciones públicas no se puede reducir a una sucesión de noticias de actualidad que aparecen y desaparecen sin tiempo para pensar sobre su significado. Es más, se ha de evitar multiplicar las noticias y anteponer la calidad a la cantidad, para no ahogar el debate público con un ruido mediático 
que sólo genera confusión y deteriora el funcionamiento democrático. Las informaciones sobre las actuaciones de los representantes políticos han de ser permanentes, ofrecer el contínuum del trabajo que se realiza, y se han de actualizar en tiempo real con datos significativos para poder evaluar el proceso de trabajo. El espacio web corporativo debe proporcionar información completa y transparente, de forma permanente, actualizada en tiempo real e inteligible, al menos sobre los siguientes elementos de la administración municipal y de la actividad de los representantes políticos que forman parte del gobierno o de la oposición:

- Los datos que permiten identificar a los representantes políticos: nombre y apellidos, fotografía, partido al que representan, biografía y o curriculum.

- Los datos que permiten conocer cómo realizan su actividad política estos representantes políticos y las decisiones que adoptan: organigrama, convocatorias de reuniones, orden del día y actas de las reuniones.

- Los datos sobre dos instrumentos básicos para poder realizar el trabajo con la mayor eficacia y eficiencia: la planificación, especialmente el Plan de Gobierno, y los presupuestos

Estas informaciones permanentes permiten fundamentar y contrastar las informaciones puntuales (noticias y comunicados) que se pueden presentar a través de la Sala de Prensa: el espacio físico y también virtual en el que se elaboran y publican las noticias y los comunicados.

4) Los recursos para la participación ciudadana efectiva

El SIC debe ofrecer en la web corporativa recursos para facilitar la participación de la ciudadanía plural. La tarea de garantizar la participación ciudadana debe estar definida al principio de cada mandato y es necesario que haya personal técnico encargado de hacerla efectiva. Esta tarea se debe contemplar en el Plan de Información y Comunicación, y su ejecución será responsabilidad del SIC.

\section{Necesidad de una Ley de Transparencia que obligue a las administraciones públicas a ofrecer información que facilite la participación ciudadana en el control democrático}

Al concluir la segunda fase del proyecto, a finales de 2011, advertimos que el punto débil de nuestro trabajo radicaba en el hecho de que en España los responsables políticos y técnicos no se interesarían por cambiar sus rutinas si no se lo exigía una Ley de Transparencia, como sucede en otros países.

El periodismo, como actividad profesional que permite transformar informaciones complejas en otras comprensibles a cualquier persona, debe colaborar a la transparencia de la gestión pública. En consecuencia, decidimos elaborar en Internet un instrumento específico para incitar a introducir mejoras en la información que proporcionan los ayuntamientos en sus webs, en la línea del Decálogo de las Buenas Prácticas de la Comunicación Pública Local. Y así fue como concebimos, en febrero de 2012, el Mapa de las Buenas Prácticas de la Comunicación Pública Local (www.infoparticipa.com/bones-practiques), que será objeto de otro artículo en cuanto nos proporcione resultados bien fundamentados. 


\section{Referencias}

BOFILL LEVI, Anna (2008): Guia per al planejament urbanistic i l'ordenació urbanística amb la incorporació de criteris de gènere. Barcelona, Generalitat de Catalunya, Institut Català de les Dones, Departament de Política Territorial i Obres Públiques.

BOFILL LEVI, Anna (2005): Planejament urbanístic, espais urbans i espais interiors des de la perspectiva de gènere. Barcelona, Generalitat de Catalunya, Institut Català de les Dones, Departament de Política Territorial i Obres Públiques; Quaderns de l'institut 6.

BRUGUÉ, Q. y GOMÀ, R. (coord.,1998): Gobierno Local y Políticas Públicas. Barcelona, Ariel.

COMISIÓN DE LAS COMUNIDADES EUROPEAS (2001): La gobernanza europea. Un Libro Blanco. (http://eur-lex.europa.eu/ [fecha de consulta: 15 de septiembre de 2012]

CORCOY RIUS, Marta (2012): Comunicació i periodisme a les corporacions locals a Catalunya (1979-2009), Tesina de doctorado. Departamento de Periodismo y Ciencias de la Comunicación. Universidad Autónoma de Barcelona: http://labcompublica.info/wp-content/uploads/2012/10/Comunicacio-i-Periodisme-a-lescorporacions-locals-a-Catalunya-1979-2009-Corcoy-2012.pdf [fecha de consulta: 30 de octubre de 2012].

CORCOY, Marta; CARRASCO, Mavi; GÁMEZ, Isabel; GÓMEZ, Patricia (2001): Les Corporacions locals i la informació pública. 1979-2001, http://labcompublica.info/wp-content/uploads/2012/09/corporacionslocals.pdf [fecha de consulta: 30 de octubre de 2012].

DE SAN MARTÍN RODRÍGUEZ, Luís Eduardo (2005): La mirada informativa de los vespertinos TAL CUAL y EL MUNDO sobre la sociedad venezolaNA (20002002). Servicio de Publicaciones de la Universidad Autónoma de Barcelona: http://www.tdx.cat/handle/10803/4183 [fecha de consulta: 15 de septiembre de 2012]

FEMENÍAS, María Luisa; SOZA ROSSI, Paula (comp.) (2011): Saberes situados / teorías trashumantes. Argentina, Facultad de Humanidades y Ciencias de la Educación de la Universidad de La Plata.

FREIXES, Teresa; REMOTTI, José Carlos (2002): El futuro de Europa: constitución $y$ derechos fundamentales. Valencia, Mínim, colección Ideas y políticas constitucionales, 3.

GENERALITAT DE CATALUNYA. DEPARTAMENT DE POLÍTICA TERRITORIAL I OBRES PÚBLIQUES (2010): Manual per a l'elaboració dels projectes d'intervenció integral en els barris i àrees urbanes d'atenció especial i per a l'elaboració de l'Informe d'Avaluació Final. Cofinanciado por el Fondo Europeo de Desarrollo Regional de la Unión Europea. Febrero de 2010 (última actualización). http://www20.gencat.cat/docs/ptop/Home/Serveis\%20i\%20tramits/Biblioteca\%20i\%20documentacio/Planificacio\%20territorial/Publicacions/Barris/Ma- 
nuals/1-Manual_Barris_2010_tcm32-24279.pdf [fecha de consulta: 15 de septiembre de 2012]

GENERALITAT DE CATALUNYA. INSTITUT CATALÀ DE LES DONES (2008): Pla de polítiques de dones de la Generalitat de Catalunya (2008-2011): http://www20.gencat.cat/portal/site/icdones/menuitem.4b491b5fa301c8a439a7264 1b0c0e1a0/?vgnextoid=00148abf9cf53210VgnVCM1000008d0c1e0aRCRD\&vgn

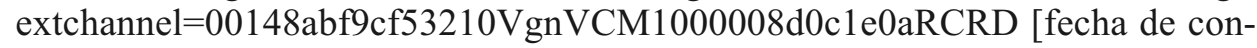
sulta: 15 de septiembre de 2012]

GENERALITAT DE CATALUNYA. INSTITUT CATALÀ DE LES DONES (2006): $V$ Plan de acción y desarrollo de las políticas de mujeres en Cataluña (2005-2007: http://www20.gencat.cat/portal/site/icdones/menuitem.4b491b5fa301c8a439a7264 1b0c0e1a0/?vgnextoid=ae727e1e45063210VgnVCM1000008d0c1e0aRCRD\&vg nextchannel=ae727e1e45063210VgnVCM1000008d0c1e0aRCRD\&vgnextfmt=d efault [fecha de consulta: 15 de septiembre de 2012]

HARAWAY, Donna J. (1995): Ciencia, cyborgs y mujeres. La reinvención de la naturaleza. Madrid, Cátedra,

JIMÉNEZ CHÁVEZ, Daniel (2012): Internet, herramienta para integrar aportaciones personales al conocimiento colectivo y la participación ciudadana. La web Histories de Barcelona (www.bdebarna.net), 2005 y 2010. Tesis Doctoral, Departamento de Periodismo de la Universidad Autónoma de Barcelona.

JIMÉNEZ CHÁVEZ, Daniel (2010): “Anotaciones espaciales y medios hiperlocales". Revista Prisma.Com, 12. Centro de Estudo das Tecnologias e Ciências da Comunicação, pp. 1-18: http://revistas.ua.pt//index.php/prismacom/article/view/753 [fecha de consulta: 15 de septiembre de 2012]

MACAGNAM, Clea Beatriz (2006): Condicionantes e implicación de revelar activos intangibles, Servicio de Publicaciones de la Universidad Autónoma de Barcelona: http://www.tesisenred.net/handle/10803/3963 [fecha de consulta: 15 de septiembre de 2012]

MARÍN OCHOA, Beatriz Elena (2009): La infografía digital, una nueva forma de comunicación. Servicio de Publicaciones de la Universidad Autónoma de Barcelona: http://www.tesisenred.net/handle/10803/48653 [fecha de consulta: 15 de septiembre de 2012]

MARTÍ-COSTA, Marc; BONET, Jordi; PYBUS, Miquel (2009): La gobernanza en los Procesos de Regeneración Urbana: La Ley de Barrios de la Generalitat de Cataluña. Institut de Govern i Politiques Públiques (IGOP), Universitat Autònoma de Barcelona: http://uab.academia.edu/JordiBonetMarti/Papers/1181114/La_gobernanza_en_los procesos_de regeneracion_urbana_La_Ley_de_Barrios_de_la Generalitat_de_Cataluna [fecha de consulta: 15 de septiembre de 2012]

MASSEY, Doreen (2004): "Lugar, identidad y geografías de la responsabilidad en un mundo en proceso de globalización". Treballs de la Societat Catalana de Geografia, 57, pp. 77-84. 
MASSEY, Doreen (1994): Space, Place and Gender. Cambridge, Polity Press.

MORENO SARDÀ, Amparo (2007): De qué hablamos cuando hablamos del hombre. Treinta años de crítica y alternativas al pensamiento androcéntrico. Barcelona, Icaria.

MORENO SARDÀ, Amparo (1998): La mirada informativa. Barcelona, Bosch.

MORENO SARDÀ, Amparo; ROVETTO GONEM, Florencia, BUITRAGO, Alfonso (2007): ¿De quién hablan las noticias? Guía para humanizar la información. Barcelona, Icaria.

MUXÍ MARTÍNEZ, Zaida (2009-a): Recomanacions per a un habitatge no jeràrquic ni androcèntric. Barcelona, Generalitat de Catalunya, Institut Català de les Dones, Departament de Medi Ambient i Habitatge, Secretaria d'Habitatge, Eines 13.

MUXÍ MARTÍNEZ, Zaida (2009-b): Urbanisme i gènere. Aplicació de la visió de gènere en la revisió de l'espai públic. Consell de les dones del districte de Sants Montjuïc. Documents de treball del Procés de Participació des dels Consells de Dones dels Districtes de Barcelona. Gener 2006-febrer 2007. II Congrés de les Dones de Barcelona. Barcelona, Ajuntament de Barcelona.

MUXÍ MARTÍNEZ, Zaida; CASANOVAS, Roser (2009): Eix temàtic 4: Les dones transformem els espais de la ciutat per a la vida quotidiana. Presentació. II Congrés de les Dones de Barcelona. Apunts pel debat. Barcelona, Ajuntament de Barcelona.

RIVERO MENÉNDEZ, José Ángel; MORA AGUDO, Leonor; FLORES UREBA, Sandra (2007): "Un estudio de la rendición de cuentas a través del e-gobierno en la administración local española". Empresa global y mercados locales: XXI Congreso Anual AEDEM. Universidad Rey Juan Carlos, Madrid, 6,7 y 8 de junio de 2007, Vol. 1, 2007 (Ponencias).

ROSE-ACKERMAN, Susan (2005): "Rendición de cuentas y estado de derecho en la consolidación de las democracias". Perfiles Latinoamericanos, $\mathrm{n}^{\mathrm{0}} 26$, julio-diciembre, México, Distrito Federal, Facultad Latinoamericana de Ciencias Sociales, pp.9-53.

ROVETTO GONEM, Florencia (2010): La representación del trabajo de las mujeres en la prensa: Análisis comparativo y cualitativo de la información de actualidad. Servicio de Publicaciones de la Universidad Autónoma de Barcelona. http://www.tdx.cat/handle/10803/4217 [fecha de consulta: 15 de septiembre de 2012]

SEGURA SORIANO, Isabel (1998): Dones de L'Hospitalet. Itineraris històrics. Hospitalet de Llobregat, Ajuntament de l'Hospitalet.

SEGURA SORIANO, Isabel (1995): Guia de dones de Barcelona. Recorreguts històrics. Barcelona, Ajuntament de Barcelona.

SIMELIO SOLÀ, Núria (2007): Prensa de información general durante la transición política (1974-1984): pervivencias y cambios en la representación de las relaciones sociales. Servicio de Publicaciones de la Universidad Autónoma de Barcelona 
http://www.tdx.cat/TDX-0327107-160534 [fecha de consulta: 15 de septiembre de 2012]

TUCHMAN, Gaye (1978): Making News: A Study in the Construction of Reality. New York, Free Press. Traducción al castellano: (1983) La producción de la noticia: estudio sobre la construcción de la realidad, Barcelona, Gustavo Gili.

VARGAS CARRILLO, María Soledad (2007): Estilos de vida, ética y estética en los dominicales de los diarios ABC, La Vanguardia y El País (1974-1999). Servicio de Publicaciones de la Universidad Autónoma de Barcelona http://www.tdx.cat/TDX0327107-161006 [fecha de consulta: 15 de septiembre de 2012].

VILLORIA MENDIETA, Manuel (2011): "La rendición de cuentas en la democracia". Temas para el debate, $\mathrm{n}^{\circ}$. 204, nov. 2011, Madrid, Fundación Sistema, pp. 32-34

VILLORIA MENDIETA, Manuel; WENCES SIMON, María Isabel (coord., 2010): Cultura de la legalidad: instituciones, procesos y estructuras. Madrid, Los Libros de la Catarata. 